PROCEEDINGS OF THE

AMERICAN MATHEMATICAL SOCIETY

Volume 130, Number 2, Pages 453-460

S 0002-9939(01)06029-4

Article electronically published on May 25, 2001

\title{
A MINIMUM ENERGY PROBLEM AND DIRICHLET SPACES
}

\author{
ANATOLII GRINSHPAN
}

(Communicated by Albert Baernstein II)

\begin{abstract}
We analyze a minimum energy problem for a discrete electrostatic model in the complex plane and discuss some applications. A natural characteristic distinguishing the state of minimum energy from other equilibrium states is established. It enables us to gain insight into the structure of positive trigonometric polynomials and Dirichlet spaces associated with finitely atomic measures. We also derive a related family of linear second order differential equations with polynomial solutions.
\end{abstract}

\section{INTRODUCTION}

The study of equilibria in electrostatic models has roots in the classical works of nineteenth century analysis (see e.g. T. J. Stieltjes [7). It is rich in applications and often helps to interpret mathematical results. One instance of this interplay is discussed in the present paper. We begin by introducing the electrostatic model.

Let $z_{1}, \ldots, z_{N}$ be $N$ fixed points in the complex plane $\mathbb{C}$ (repetitions allowed) each bearing a positive unit charge. Suppose that none of them is zero or lies on the unit circumference $\mathbb{T}=\{z:|z|=1\}$. Furthermore, let $e^{i \theta_{1}}, \ldots, e^{i \theta_{N}}$ be the positions of $N$ movable particles of negative unit charge, restrained to $\mathbb{T}$.

Assume that these electrostatic masses interact according to the law of logarithmic potential, i.e. the charge of strength $q_{1}$ at the point $a$ acts on the charge of strength $q_{2}$ at the point $b$ with the force $2 q_{1} q_{2} / \overline{(b-a)}$. We interpret the function

$$
\mathcal{I}\left(e^{i \theta_{1}}, \ldots, e^{i \theta_{N}}\right)=\sum_{\substack{k, n=1 \\ k<n}}^{N} \log \frac{1}{\left|e^{i \theta_{n}}-e^{i \theta_{k}}\right|}-\sum_{k, n=1}^{N} \log \frac{1}{\left|e^{i \theta_{n}}-z_{k}\right|}
$$

as the discrete energy of the system. We denote this system by $S(N, N)$ (see Figure 1).

Note that the particles positioned at $\lambda_{1}, \ldots, \lambda_{N}$ on $\mathbb{T}$ are in equilibrium if and only if the resultant electrostatic force at each of them is normal to the circle, i.e. for each $n=1, \ldots, N$

$$
\Im\left\{\lambda_{n}\left[\sum_{k \neq n} \frac{1}{\lambda_{n}-\lambda_{k}}-\sum_{k=1}^{N} \frac{1}{\lambda_{n}-z_{k}}\right]\right\}=0 .
$$

Received by the editors January 18, 2000 and, in revised form, June 22, 2000.

2000 Mathematics Subject Classification. Primary 31A99, 46E20, 78A30, 31A35.

Key words and phrases. Electrostatic equilibrium, Dirichlet spaces, Lamé differential equation.

(C)2001 American Mathematical Society 


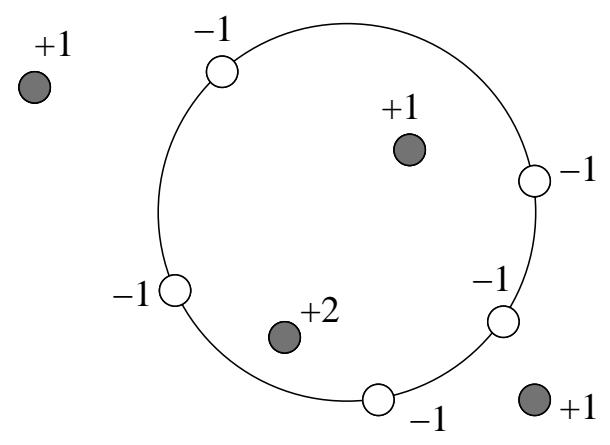

Figure 1. A system $S(5,5)$

Equivalently, $\left(\lambda_{1}, \ldots, \lambda_{N}\right)$ is a critical point for the energy function $\mathcal{I}$, so that the vanishing of the gradient,

$$
\nabla \mathcal{I}=0
$$

is the condition of equilibrium in $S(N, N)$.

In Section 2 we show that every system $S(N, N)$ admits a unique configuration $\lambda_{1}, \ldots, \lambda_{N}$ minimizing $\mathcal{I}$. This unique point of minimum satisfies the equality of mean arguments, $\prod_{n=1}^{N} z_{n} / \prod_{n=1}^{N} \lambda_{n}>0$, and for any other equilibrium configuration we have $\prod_{n=1}^{N} z_{n} / \prod_{n=1}^{N} \lambda_{n}<0$. The mean argument property follows from a critical point analysis of the energy function $\mathcal{I}$. To show that a minimum is unique we adopt an argument of D. Sarason and D. Suarez [6], used to prove the uniqueness of a solution to a certain inverse problem (see Theorem 2, Section 3 below).

In Sections 3 and 4 we discuss some closely related facts about discrete Dirichlet spaces and polynomial solutions to the Lamé differential equation.

A notion of the polynomial inverse will be used throughout the paper. Given a polynomial $p(z)$ of degree $N$ its polynomial inverse is defined by $p^{*}(z)=z^{N} \overline{p(1 / \bar{z})}$. It has zeros symmetric to those of $p$ with respect to $\mathbb{T}$.

\section{The MAIN RESULT}

We now formulate and prove our central statement.

Theorem 1. Every electrostatic system $S(N, N)$ has a unique configuration of minimum energy (the ground state) $\lambda_{1}, \ldots, \lambda_{N}$ on the unit circle $\mathbb{T}$. This ground state obeys equality of average arguments

$$
\frac{z_{1} \cdot \ldots \cdot z_{N}}{\lambda_{1} \cdot \ldots \cdot \lambda_{N}}>0
$$

and varies continuously with $z_{1}, \ldots, z_{N}$ for $z_{n} \notin \mathbb{T} \cup\{0\}, n=1, \ldots, N$. Any other equilibrium configuration $\lambda_{1}, \ldots, \lambda_{N}$ of the system is unstable and satisfies

$$
\frac{z_{1} \cdot \ldots \cdot z_{N}}{\lambda_{1} \cdot \ldots \cdot \lambda_{N}}<0
$$

Proof. One readily verifies the above statements in the case $N=1$. Henceforth we assume that $N \geq 2$. 
Let $p(z)=\prod_{n=1}^{N}\left(z-z_{n}\right)$ be the monic polynomial with zeros at the positions of fixed positive charges. Write the energy function of $S(N, N)$ as

$$
\mathcal{I}\left(e^{i \theta_{1}}, \ldots, e^{i \theta_{N}}\right)=\Re\left\{\log \frac{p\left(e^{i \theta_{1}}\right) \cdot \ldots \cdot p\left(e^{i \theta_{N}}\right)}{V\left(e^{i \theta_{1}}, \ldots, e^{i \theta_{N}}\right)}\right\}
$$

where

$$
V\left(x_{1}, \ldots, x_{N}\right)=\prod_{1 \leq m<n \leq N}\left(x_{n}-x_{m}\right)
$$

is the Vandermonde determinant. Since $\mathcal{I}$ maps the torus $\mathbb{T}^{N}$ continuously into $(-\infty, \infty]$ it attains a global minimum, which establishes the existence of the ground state.

Consequently the following system of $N$ equations is satisfied by some $N$-tuple of pairwise distinct points on $\mathbb{T}$ :

$$
\frac{\partial \mathcal{I}}{\partial \theta_{n}}=\Im\left\{\sum_{k \neq n} \frac{e^{i \theta_{n}}}{e^{i \theta_{n}}-e^{i \theta_{k}}}-\sum_{k=1}^{N} \frac{e^{i \theta_{n}}}{e^{i \theta_{n}}-z_{k}}\right\}=0, \quad n=1, \ldots, N .
$$

Now let $\left(\lambda_{1}, \ldots, \lambda_{N}\right)$ be any collection of pairwise distinct points on $\mathbb{T}$ satisfying (3) and let $\varphi(z)=\prod_{n=1}^{N}\left(z-\lambda_{n}\right)$. Introduce the self-inversive polynomials

$$
P(z)=p(z) p^{*}(z)
$$

and

$$
S(z)=z \varphi(z) \varphi^{*}(z) \sum_{n=1}^{N}\left|c_{n}\right|^{2}\left[\left(z-\lambda_{n}\right)\left(1-\bar{\lambda}_{n} z\right)\right]^{-1},
$$

where $c_{n}=p\left(\lambda_{n}\right) / \varphi^{\prime}\left(\lambda_{n}\right), n=1, \ldots, N$. Then

$$
\begin{gathered}
z \frac{P^{\prime}(z)}{P(z)}=N+2 i \Im\left\{\sum_{n=1}^{N} \frac{z}{z-z_{n}}\right\}, \quad|z|=1, \\
\lambda_{n} \frac{S^{\prime}\left(\lambda_{n}\right)}{S\left(\lambda_{n}\right)}=N+2 i \Im\left\{\sum_{k \neq n} \frac{\lambda_{n}}{\lambda_{n}-\lambda_{k}}\right\}, \quad n=1, \ldots, N .
\end{gathered}
$$

By the choice of the coefficients $c_{n}$ and in light of (3), the polynomials $P(z)$ and $S(z)$ agree together with their derivatives at each point $\lambda_{n}, n=1, \ldots, N$. As a result

$$
P(z)-S(z)=c \varphi(z) \varphi^{*}(z)
$$

for some complex number $c \neq 0$. Setting $z=0$ we find that $c=p(0) / \varphi(0)$. For $z$ on $\mathbb{T}$ the last identity can be written as

$$
\left|\frac{p(z)}{\varphi(z)}\right|^{2}=c+\sum_{n=1}^{N}\left|\frac{c_{n}}{z-\lambda_{n}}\right|^{2}, \quad|z|=1 .
$$

Clearly $c=\prod_{n=1}^{N} z_{n} / \prod_{n=1}^{N} \lambda_{n}$ is real. Note that (4) and (3) are equivalent.

To confirm (1) it suffices to show that $c$ is positive if $\mathcal{I}$ attains a local minimum at $\left(\lambda_{1}, \ldots, \lambda_{N}\right)$. To see this write

$$
v_{n}(z)=\left(z-\lambda_{n}\right) \frac{p(z)}{\varphi(z)}, \quad n=1, \ldots, N .
$$


Then $v_{n}\left(\lambda_{n}\right)=c_{n}$. Furthermore,

$$
\begin{gathered}
\frac{\partial \mathcal{I}}{\partial \theta_{n}}\left(\lambda_{1}, \ldots, \lambda_{N}\right)=\left.\frac{\partial}{\partial \theta} \log \left|v_{n}\left(e^{i \theta}\right)\right|\right|_{\lambda_{n}}=0, \\
\mathcal{I}_{n n}=\frac{\partial^{2} \mathcal{I}}{\partial \theta_{n}^{2}}\left(\lambda_{1}, \ldots, \lambda_{N}\right)=\left.\frac{\partial^{2}}{\partial \theta^{2}} \log \left|v_{n}\left(e^{i \theta}\right)\right|\right|_{\lambda_{n}},
\end{gathered}
$$

and using l'Hôpital's rule and (4) we conclude that

$$
\left|c_{n}\right|^{2} \mathcal{I}_{n n}=\lim _{e^{i \theta} \rightarrow \lambda_{n}} \frac{\left|v_{n}\left(e^{i \theta}\right)\right|^{2}-\left|c_{n}\right|^{2}}{\left|e^{i \theta}-\lambda_{n}\right|^{2}}=c+\sum_{k \neq n}\left|\frac{c_{k}}{\lambda_{n}-\lambda_{k}}\right|^{2} .
$$

Also, directly from the definition of $\mathcal{I}$, for $k \neq n$,

$$
\mathcal{I}_{k n}=\frac{\partial^{2} \mathcal{I}}{\partial \theta_{k} \partial \theta_{n}}\left(\lambda_{1}, \ldots, \lambda_{N}\right)=\frac{-1}{\left|\lambda_{n}-\lambda_{k}\right|^{2}} .
$$

Consequently, the following identity holds:

$$
\sum_{k, n=1}^{N} \mathcal{I}_{k n}\left|c_{k}\right|^{2}\left|c_{n}\right|^{2}=c \sum_{n=1}^{N}\left|c_{n}\right|^{2}
$$

In particular, if $\left(\lambda_{1}, \ldots, \lambda_{N}\right)$ is a point of minimum, the matrix $\left(\mathcal{I}_{k n}\right)_{k, n=1}^{N}$ is positive semidefinite and so $c$ must be positive.

To prove that the energy function attains but one minimum on $\mathbb{T}^{N}$ we make use of an argument from [6]. In fact, (3) has a unique solution satisfying (1). For otherwise we infer from (4) that for some $p(z)=\prod_{n=1}^{N}\left(z-z_{n}\right)$, with $\left|z_{n}\right| \neq 0,1$, there exist two distinct polynomials $\varphi(z)=\prod_{n=1}^{N}\left(z-\lambda_{n}\right)$ and $\tilde{\varphi}(z)=\prod_{n=1}^{N}\left(z-\tilde{\lambda}_{n}\right)$, with $\left|\lambda_{n}\right|=\left|\tilde{\lambda}_{n}\right|=1$, such that for $|z|=1$

$$
|\varphi(z)|^{2}\left(|p(0)|+\sum_{n=1}^{N} \frac{a_{n}}{\left|z-\lambda_{n}\right|^{2}}\right)=|\tilde{\varphi}(z)|^{2}\left(|p(0)|+\sum_{n=1}^{N} \frac{\tilde{a}_{n}}{\left|z-\tilde{\lambda}_{n}\right|^{2}}\right),
$$

where $a_{n}, \tilde{a}_{n}$ are positive numbers. Since

$$
\left|\frac{\varphi(z)}{z-\lambda_{n}}\right|^{2}, \quad\left|\frac{\tilde{\varphi}(z)}{z-\tilde{\lambda}_{n}}\right|^{2}, \quad n=1, \ldots, N,
$$

are linearly dependent real trigonometric polynomials on $\mathbb{T}$ there are real numbers $x_{n}, y_{n}$ (not all zero) such that for $|z|=1$

$$
|\varphi(z)|^{2}\left(|p(0)|+\sum_{n=1}^{N} \frac{a_{n}+t x_{n}}{\left|z-\lambda_{n}\right|^{2}}\right)=|\tilde{\varphi}(z)|^{2}\left(|p(0)|+\sum_{n=1}^{N} \frac{\tilde{a}_{n}+t y_{n}}{\left|z-\tilde{\lambda}_{n}\right|^{2}}\right),
$$

where $t$ is a real parameter. When $|t|$ is small the numbers $a_{n}+t x_{n}, \tilde{a}_{n}+t y_{n}$ are all positive. Choose $t$ of the smallest absolute value so that at least one of them vanishes. Then both sides of the last identity must have at least one common factor of the form $\left|z-\lambda_{n}\right|^{2}$, which can therefore be removed. Repeating this procedure if necessary we conclude after finitely many steps that $\varphi(z)=\tilde{\varphi}(z)$, contradicting the assumption.

Finally we address the continuity of the ground state on the parameters. Let $\mathbf{z}=\left(z_{1}, \ldots, z_{N}\right) \in \mathbb{C}^{N}$ and $\boldsymbol{\lambda}=\left(e^{i \theta_{1}}, \ldots, e^{i \theta_{N}}\right) \in \mathbb{T}^{N}$. View $f(\mathbf{z}, \boldsymbol{\lambda})=e^{-\mathcal{I}}$ as a 
function on $\mathbb{G}^{N} \times \mathbb{T}^{N}$, where $\mathbb{G}=\{z \in \mathbb{C}:|z| \neq 0,1\}$. Clearly $f$ is continuous and, as we have proved, for each $\mathbf{z} \in \mathbb{G}^{N}$ there is a unique $\boldsymbol{\lambda}_{\mathbf{z}} \in \mathbb{T}^{N}$ such that

$$
f\left(\mathbf{z}, \boldsymbol{\lambda}_{\mathbf{z}}\right)=\max _{\boldsymbol{\lambda} \in \mathbb{T}^{N}} f(\mathbf{z}, \boldsymbol{\lambda}) .
$$

It is easy to see that $f\left(\mathbf{z}, \boldsymbol{\lambda}_{\mathbf{z}}\right)$ is continuous for $\mathbf{z} \in \mathbb{G}^{N}$. Hence, by uniqueness of the point of maximum, we conclude that the function $\mathbf{z} \mapsto \boldsymbol{\lambda}_{\mathbf{z}}$ is also continuous on $\mathbb{G}^{N}$. The proof of Theorem 1 is complete.

\section{Application to Dirichlet spaces}

Theorem 1 provides a natural approach to the following theorem proved by Sarason and Suarez and used by Sarason in his study of the Dirichlet spaces associated with finitely atomic measures $[5]$. We denote the unit disk by $\mathbb{D}$ and let $K(z)=z /(1-z)^{2}$.

Theorem 2 ([6]). Let $z_{1}, \ldots, z_{N}$ be a finite sequence of points in $\mathbb{D} \backslash\{0\}$ (repetitions allowed). Then there is a unique finitely atomic positive measure $\mu$ on $\mathbb{T}$, consisting of $N$ atoms, such that $z_{1}, \ldots, z_{N}$ is the zero sequence in $\mathbb{D}$ of the function

$$
\mathcal{K}_{\mu}(z)=1-\int_{\mathbb{T}} K(\bar{\lambda} z) d \mu(\lambda) .
$$

Now that the equilibrium states of $S(N, N)$ have been analyzed, we can obtain Theorem 2 as a direct consequence of Theorem 1.

Proof. Define the polynomial $p(z)=\prod_{n=1}^{N}\left(z-z_{n}\right)$. Theorem 2, in fact, asserts that there is a unique positive measure $\mu=\sum_{n=1}^{N} \mu_{n} \delta_{\lambda_{n}}$ such that

$$
\frac{1}{c} \frac{p(z) p^{*}(z)}{\varphi(z) \varphi^{*}(z)}=1-\sum_{n=1}^{N} \mu_{n} K\left(\bar{\lambda}_{n} z\right)
$$

where $\varphi(z)=\prod_{n=1}^{N}\left(z-\lambda_{n}\right)$ and $c=p(0) / \varphi(0)$. Equivalently, the positive trigonometric polynomial $\left|p\left(e^{i \theta}\right)\right|^{2}$ can be uniquely represented as

$$
\left|p\left(e^{i \theta}\right)\right|^{2}=c\left|\varphi\left(e^{i \theta}\right)\right|^{2}\left(1+\sum_{n=1}^{N} \frac{\mu_{n}}{\left|e^{i \theta}-\lambda_{n}\right|^{2}}\right), \quad c=|p(0)|,
$$

where $\lambda_{n} \in \mathbb{T}, n=1, \ldots, N$. Comparing (5) and (4) we restate the last assertion: the function $\mathcal{I}$ defined by (2) has a unique critical point $\left(\lambda_{1}, \ldots, \lambda_{N}\right) \in \mathbb{T}^{N}$ with $c=\prod_{n=1}^{N} z_{n} / \prod_{n=1}^{N} \lambda_{n}>0$. By Theorem 1 this is the case and the unique measure sought is given by

$$
\mu=\sum_{n=1}^{N} \mu_{n} \delta_{\lambda_{n}}, \quad \mu_{n}=\frac{1}{c}\left|\frac{p\left(\lambda_{n}\right)}{\varphi^{\prime}\left(\lambda_{n}\right)}\right|^{2}
$$

where $\left(\lambda_{1}, \ldots, \lambda_{N}\right)$ minimizes $\mathcal{I}$. The proof of Theorem 2 is complete.

We now provide some background to explain the connection with Dirichlet spaces. A detailed discussion can be found in the papers of S. Richter [4] and Sarason [5]. Given a finite positive Borel measure $\mu$ on $\mathbb{T}$, one can construct its Poisson integral $\mathrm{P} \mu$, a function harmonic and positive in $\mathbb{D}$ :

$$
\mathrm{P} \mu(r, \theta)=\frac{1}{2 \pi} \int_{0}^{2 \pi} \mathrm{P}(r, \theta-t) d \mu(t)
$$


Here $\mathrm{P}(r, \theta)=\Re\left\{\left(1+r e^{i \theta}\right) /\left(1-r e^{i \theta}\right)\right\}$ is the Poisson kernel. The harmonically weighted Dirichlet space $D(\mu)$ is then defined to be the space of all functions $f$, analytic in $\mathbb{D}$, which possess a finite Dirichlet integral

$$
D_{\mu}(f)=\iint_{\mathbb{D}}\left|f^{\prime}\right|^{2} \mathrm{P} \mu d \sigma<\infty,
$$

where $\sigma$ is normalized area measure.

One can show that $D(\mu)$ is always contained in the Hardy space $H^{2}(\mathbb{D})$. If $\mu$ is normalized arclength, $D(\mu)$ is the classical Dirichlet space of analytic functions in $\mathbb{D}$ whose derivatives are square integrable over $\mathbb{D}$.

For $f \in D(\mu)$ one defines its norm $\|f\|_{\mu}$ by

$$
\|f\|_{\mu}^{2}=\|f\|_{H^{2}}^{2}+D_{\mu}(f) \text {. }
$$

The corresponding inner product $\langle\cdot, \cdot\rangle_{\mu}$, which makes $D(\mu)$ into a Hilbert space, is given by

$$
\langle f, g\rangle_{\mu}=\langle f, g\rangle_{H^{2}}+\iint_{\mathbb{D}} f^{\prime} \overline{g^{\prime}} \mathrm{P} \mu d \sigma .
$$

Restricting to the case when $\mu$ is finitely atomic, $\mu=\sum_{n=1}^{N} \mu_{n} \delta_{\lambda_{n}}$, we observe that the connection between the points $\left\{z_{n}\right\}_{n=1}^{N}$ and $\left\{\lambda_{n}\right\}_{n=1}^{N}$ that underlies Theorem 2 plays an important role in the study of $D(\mu)$. It has been shown by Sarason 5] that $D(\mu)$ is actually the orthogonal direct sum of two subspaces,

$$
D(\mu)=\varphi H^{2} \oplus \mathcal{H}_{p}
$$

where $\varphi H^{2}=\left\{\varphi f: f \in H^{2}\right\}$ is isometric to $H^{2}$ and $\mathcal{H}_{p}$ is the $N$-dimensional space of rational functions of the form $s(z) / p^{*}(z)$ with $s(z)$ a polynomial of degree less than $N$. Furthermore, Sarason's analysis of wandering vectors of the shift operator on $D(\mu)$ extensively uses representations of type (5).

Note that orthogonal decomposition (6) allows one to formulate the following "discrete" version of Dirichlet principle: the unique minimum of $\|f\|_{\mu}$ taken over all $f \in D(\mu)$ with fixed boundary values $f\left(\lambda_{n}\right)=w_{n}, n=1, \ldots, N$, is furnished by a rational function.

Indeed, there is a unique rational function $r(z)$ in $\mathcal{H}_{p}$ satisfying these boundary conditions, and for any admissible $f$ one has

$$
\|f\|_{\mu}^{2}=\|f-r\|_{\mu}^{2}+\|r\|_{\mu}^{2}>\|r\|_{\mu}^{2}
$$

unless $f=r$ in $D(\mu)$.

\section{Equations of Lamé AND their polynomial solutions}

The search for polynomial solutions of second order linear differential equations and electrostatic interpretation of their zeros go back to E. Heine [2] and Stieltjes 7]. Consider the generalized Lamé equation

$$
A(z) w^{\prime \prime}(z)+B(z) w^{\prime}(z)+C(z) w(z)=0,
$$

where $A(z)$ is a polynomial of degree $(\nu+1)$ and $B(z)$ is a polynomial of degree $\nu$. It is often of interest to find a polynomial $C(z)$ of degree at most $(\nu-1)$ such that the equation admits a polynomial solution $w(z)$ of given degree $N$. The zeros of such $w(z)$ can be viewed as an equilibrium state of a system of charges in the presence of external field. These questions have been well-studied in several important cases. 
See the monographs by G. Szegö [8] and M. Marden [3] for a background, and the work of F. A. Grünbaum [1] who considered a model with charges of both signs on the interval $[-1,1]$.

For every system $S(N, N)$ a polynomial $\varphi(z)=\prod_{n=1}^{N}\left(z-\lambda_{n}\right)$ with zeros in equilibrium can be seen to satisfy Lamé's differential equation. Indeed, let $p(z)=$ $\prod_{n=1}^{N}\left(z-z_{n}\right)$. Using the identities

$$
\frac{\varphi^{\prime \prime}\left(\lambda_{n}\right)}{\varphi^{\prime}\left(\lambda_{n}\right)}=2 \sum_{k \neq n} \frac{1}{\lambda_{n}-\lambda_{k}}, \quad n=1, \ldots, N
$$

and noting that for $\lambda \in \mathbb{T}$

$$
\Re\left\{\frac{\lambda}{\lambda-z}\right\}=\frac{1}{2}\left(1+\frac{1-|z|^{2}}{|\lambda-z|^{2}}\right) \quad \text { and } \quad \frac{\lambda}{\lambda-z}+\frac{\bar{z} \lambda}{1-\bar{z} \lambda}=\frac{1-|z|^{2}}{|\lambda-z|^{2}},
$$

one checks that the equilibrium condition (3) implies that for each $n=1, \ldots, N$

$$
\frac{1}{2} \lambda_{n} \frac{\varphi^{\prime \prime}\left(\lambda_{n}\right)}{\varphi^{\prime}\left(\lambda_{n}\right)}-\lambda_{n} \frac{p^{\prime}\left(\lambda_{n}\right)}{p\left(\lambda_{n}\right)}=-\frac{1}{2}\left(1+\lambda_{n} \frac{p^{\prime}\left(\lambda_{n}\right)}{p\left(\lambda_{n}\right)}-\lambda_{n} \frac{p^{* \prime}\left(\lambda_{n}\right)}{p^{*}\left(\lambda_{n}\right)}\right) .
$$

Consequently the function

$$
z \frac{\varphi^{\prime \prime}(z)}{\varphi^{\prime}(z)}-z\left(\frac{p^{\prime}(z)}{p(z)}+\frac{p^{* \prime}(z)}{p^{*}(z)}\right)+1
$$

vanishes at each point $z=\lambda_{n}$ and so there is a polynomial $C(z)$ of degree at most $(2 N-1)$ such that $w=\varphi(z)$ satisfies the equation

$$
z P(z) w^{\prime \prime}(z)+\left[P(z)-z P^{\prime}(z)\right] w^{\prime}(z)+C(z) w(z)=0,
$$

where $P(z)=p(z) p^{*}(z)$.

According to Theorem 1 for every $p(z)=\prod_{n=1}^{N}\left(z-z_{n}\right)$ with $\left|z_{n}\right| \neq 0,1$ there exists a unique polynomial $C(z)$ such that (7) is satisfied by an $N$-th degree polynomial $w=\varphi(z)$ with zeros minimizing the energy of $S(N, N)$. In this case $p(0) / \varphi(0)>0$. For any polynomial $C(z)$ such that $(7)$ has a polynomial solution $w=\varphi(z)$ associated with a different equilibrium of $S(N, N)$, the ratio $p(0) / \varphi(0)$ is negative.

By way of illustration we take the points $z_{1}, \ldots, z_{N}$ to be equidistributed on the circle of radius $r>0$. Then the configuration of minimum energy is given by the $N$-th roots of unity. Thus $p(z)=z^{N}-r^{N}, P(z)=\left(z^{N}-r^{N}\right)\left(1-r^{N} z^{N}\right)$, $\varphi(z)=z^{N}-1$, and $C(z)=-N^{2} r^{N} z^{N-1}\left(z^{N}+1\right)$. Another equilibrium configuration is realized by the zeros of $w=z^{N}+1$; the corresponding coefficient in (7) is $C(z)=-N^{2} r^{N} z^{N-1}\left(z^{N}-1\right)$.

One can consider more general systems. For instance, if $\omega=\sum_{m=1}^{M} \omega_{m} \delta_{z_{m}}$ is a finitely atomic real measure with points of positive mass not on $\mathbb{T}$, we define $S(\omega, N)$ by analogy with $S(N, N)$. Every such system has at least one equilibrium configuration (the ground state) and it is easy to write down the Lamé equation satisfied by a polynomial $w=\varphi(z)$ with zeros given by an equilibrium of $S(\omega, N)$.

\section{REFERENCES}

1. F. A. Grünbaum, Variations on a theme of Heine and Stieltjes: an electrostatic interpretation of the zeros of certain polynomials, J. Comput. Appl. Math. 99 (1998), 189-194. MR 99j:33012

2. E. Heine, Handbuch der Kugelfunctionen, Bd I, J. Springer, Berlin, 1878. MR 34:4564

3. M. Marden, Geometry of Polynomials, Amer. Math. Soc., Providence, RI, 1989. 
4. S. Richter, A representation theorem for cyclic analytic two-isometries, Trans. Amer. Math. Soc., 328 (1991), 325-349. MR 92e:47052

5. D. Sarason, Harmonically weighted Dirichlet spaces associated with finitely atomic measures, Integral Equations and Operator Theory, 31 (1998), 186-213; Errata, 36 (2000), no. 4, 499-504. MR 99i:46015 CMP 2000:13

6. D. Sarason, D. Suarez, Inverse problem for the zeros of certain Koebe-related functions, Journal d'Analyse Mathematique, 71 (1997), 149-158. MR 98g:30009

7. T. J. Stieltjes, Sur certains polynômes qui vérifient une équation différentielle linéaire du second ordre et sur la théorie des fonctions de Lamé, Acta Math. 8 (1885), 321-326; Oeuvres Complètes 1, 434-439.

8. G. Szegö, Orthogonal polynomials, Fourth Edition, Amer. Math. Soc., Providence, RI, 1975. MR 51:8724

Department of Mathematics, University of California, Berkeley, California 94720

E-mail address: tolya@math.berkeley.edu 